\title{
Patient and Care Delays of Breast Cancer in China
}

\section{Yue-Lin Li, MD' \\ Ya-Chao Oin, MS ${ }^{1}$ \\ Lu-Ying Tang, MD2 \\ Yu-Huang Liao, MS'1 \\ Wei Zhang, MS ${ }^{1}$ \\ Xiao-Ming Xie, MD, $\mathrm{PhD}^{3}$ \\ Oiang Liu, MD, PhD ${ }^{4}$ \\ Ying Lin, $\mathrm{MD}, \mathrm{Ph} \mathrm{D}^{5}$ \\ Ze-Fang Ren, $\mathrm{MD}, \mathrm{PhD}^{1}$}

\section{Purpose}

This study differentiates patient and care delays of breast cancer and explores the related factors as well as the associations with the prognosis in Guangzhou, a southern city of China.

\section{Materials and Methods}

A cohort of female incident breast cancer patients $(n=1,551)$ was recruited from October 2008 to March 2012 and followed up until January 1, $2016(n=1,374)$ in the affiliated hospitals of Sun Yat-sen University. The factors associated with patient and care delays were analyzed with multivariable logistic models. Cox proportional hazards regression models were constructed to estimate the impacts of the delays on the prognosis.

\section{Results}

There were $40.4 \%$ patient delay ( $\geq 3$ months) and $15.5 \%$ care delay ( $\geq 1$ month). The patient delay, but not the care delay, was significantly related to the clinical stage and consequently worsened the prognosis of breast cancer (hazard ratio, 1.45; 95\% confidence interval, 1.09 to 1.91 for progression-free survival). The factors related to an increased patient delay included premenopausal status, history of benign breast disease, and less physical examination.

\section{Conclusion}

Patient delay was the main type of delay in Guangzhou and resulted in higher clinical stage and poor prognosis of breast cancer. Screening for breast cancer among premenopausal women may be an effective way to reduce this delay.

\section{Key words}

Breast neoplasms, Patient delay, Care delay, Related factors, Prognosis
Correspondence: Ze-Fang Ren, MD, PhD Department of Statistics and Epidemiology, School of Public Health, Sun Yat-sen University, 74 Zhongshan 2nd Rd, Guangzhou 510080, China Tel: 86-2087332577

Fax: 86-2087330678

E-mail: renzef@mail.sysu.edu.cn

Received July 2, 2018

Accepted November 3, 2018

Published Online November 6, 2018

*Yue-Lin Li and Ya-Chao Qin contributed equally to this work. 


\section{Introduction}

Breast cancer is one of the major health concerns for females in the world. Delaying diagnosis or treatment is likely to result in tumor progression and worse prognosis. The delays were usually divided into patient delay (the period from first onset of symptoms or signs to first medical consultation) and care delay (the period from first consultation to definite treatment) [1]. These two types of delays resulted in divergent consequences: patient delay was consistently found to be associated with a higher clinical stage and a lower survival rate [2,3], while the effects of care delay were complex and obscure [4-9]. However, these studies were mostly conducted in developed countries. It was known that people in developed areas had more knowledge of breast cancer and would have a shorter patient delay, while they may have a longer care delay due to the outpatient appointment system [10]. In China and some other developing areas, contrarily, people had relatively poor knowledge of breast cancer and may have a longer patient delay, while patients would be diagnosed and treated efficiently once they sought medical consultation $[11,12]$.

There was one related study in China [13], but the patient and care delays were not separated and it was unable to compare the delays with other studies. Moreover, the factors predicting delay in this previous study did not include socioeconomic status which was the important predictors of patient and care delays $[14,15]$. Moreover, that study did not consider estrogen receptor (ER), progesterone receptor (PR), and human epidermal growth factor receptor 2 (HER2), which are key clinicopathologic characteristics for prognosis of breast cancer.

We therefore conducted a follow-up study using a breast cancer population in Guangzhou, China (GZBCS), in order to (1) obtain the incidences of both patient and care delays; (2) explore the factors affecting the two delays; (3) examine the relationships between the delays and clinicopathologic features; and (4) evaluate the associations of the delays with the prognosis of breast cancer.

\section{Materials and Methods}

\section{Study population}

A total of 1,551 female patients pathologically diagnosed with incident primary breast cancer were recruited from the First and the Second Affiliated Hospitals and the Cancer Center of Sun Yat-sen University in Guangzhou, China, from

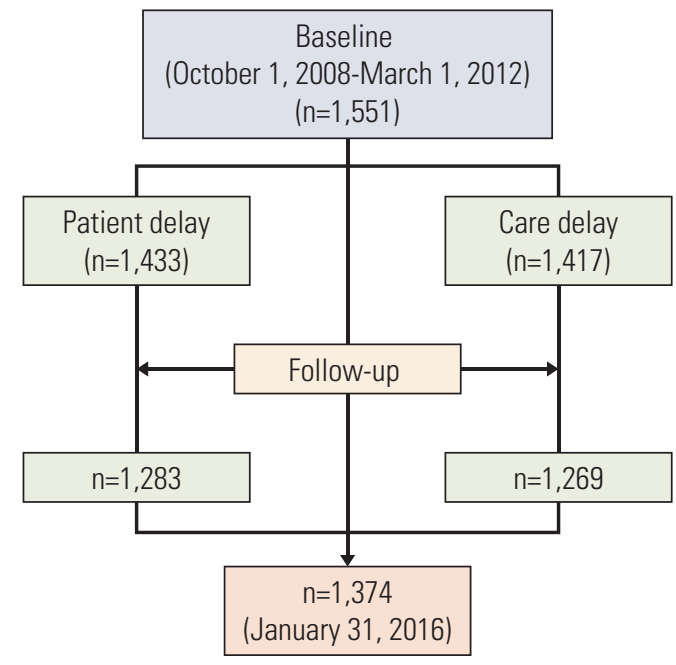

Fig. 1. Patient numbers in baseline and successfully follow-up corresponding to patient and care delays.

October 2008 to March 2012. Women with a history of any cancers were excluded.

\section{Data collection and variable definitions}

The demographic data were obtained through face to face interview by trained interviewers using a structured questionnaire, including age, education, residential status, family monthly income, menopausal status, age at menarche, comorbidities, history of breast disease/pregnant/parity, family history of breast cancer, history of night shift, and health examination. Height and weight were measured by the nurses upon admission to the hospital. Clinical characteristics of breast cancer patients were collected from medical records and pathological reports. The statuses of ER, PR, and HER2 of breast cancer tissues were determined by pathologists using immunohistochemical tests. The definitions of ER, PR, and HER2 statuses were previously described in detail [16]. The information about the initial sign or symptom and the corresponding time was also achieved by reviewing the medical records. The first sign or symptom included "selfdetected" (the patient or a family member/friend detected a lump, nipple change, or other breast abnormity), "examdetected" (a health care provider detected an abnormality), "imaging-detected" (a mammogram, breast magnetic resonance image, or other imaging test indicated an abnormality). We defined patient delay as at least 3 months from either the first sign or symptom to seeking medical attention and care delay as at least 1 month from seeking medical attention to receiving therapy. There were 1,433 and 1,417 patients who had successfully been collected the information about 
patient delay and care delay, respectively (Fig. 1).

\section{Follow-up}

The patients were followed up at least every 3 months during the first year and every 6 months during the second and the third year after treatment; thereafter, patients were followed up once every year until death or January 31, 2016. The means of follow-up included outpatient visit, phone call, and correspondence. The median follow-up duration was 57.3 months. The follow-up data were obtained from 1,374 breast cancer patients (patients delay 1,283 [89.5\%], care delay 1,269 [89.6\%]) (Fig. 1). The primary endpoint for this study was overall survival (OS), defined as the time from diagnosis until death; the patients still alive have been censored at their latest date of follow-up. The second endpoint was progression-free survival (PFS), calculated from diagnosis to the date of progression (including recurrence, metastasis, or death); the patients still alive without progression have been censored at the latest date of their follow-up.

\section{Statistical analysis}

To evaluate the possible predictors influencing patient and care delays of breast cancer, we conducted univariate and multivariate analyses with each of explanatory variables, including age, education, residence, family monthly income, menopausal status, age at menarche, pregnancy, parity, breastfeeding, body mass index (BMI), family history of breast cancer, history of benign breast disease, comorbidities, physical examination. Potential confounders were brought into the multivariate logistic analyses. The associations between delays and clinicopathologic features of breast cancer, such as clinical stage, ER, PR, and HER2 status, were also assessed using the same models. Odds ratios (ORs) and 95\% confidence intervals (CIs) were estimated to evaluate these relationships.

We assessed the associations of patient/care delays with the prognosis using the Cox proportional hazards model adjusted for the potential confounders, including age, education, BMI, family history of breast cancer, clinical stage, ER status, and HER2 status. A sensitivity analysis was performed by including or excluding clinical stage or ER status in the model. Hazard ratios (HRs) and 95\% CIs were estimated to assess the associations. All statistical tests were twotailed with $p<0.05$ considered to be significant. All the analyses above were conducted by SPSS Statistics software, ver. 23.0 (IBM Corp., Armonk, NY).

\section{Ethical statement}

All patients were asked to provide informed consent before inclusion. The study was approved by the Ethical Committee of the School of Public Health in Sun Yat-sen University.

\section{Results}

\section{Patterns of delays}

The initial signs or symptoms for the majority of the patients $(93.6 \%)$ were self-detected breast abnormity, and a few patients were exam-detected $(5.6 \%)$ or imaging-detected $(0.8 \%)$. Forty percent of the women had $>3$ months of patient delay, and the median time was 2 months (25th to 75th percentile, 0.5 to 10.0 months). Only $15.5 \%$ of the women had $>1$ month of care delay and the median time was 0.37 months (25th to 75 th percentile, 0.20 to 0.67 months). The percentage of patients experiencing the total delay (a combination of patient and care delays) of longer than 1, 3, and 6 months were $75.1 \%, 51.0 \%$, and $37.4 \%$, respectively; the median time was 3.18 months (25th to 75 th percentile, 1.07 to 12.17 months). The details of the delays were shown in Table 1.

Table 1. Types of the delays of breast cancer and the rates in Guangzhou, China

\begin{tabular}{|c|c|c|c|c|c|}
\hline Type of delay & $\begin{array}{l}\text { Total No. of } \\
\text { responding patients }\end{array}$ & > 1 Month & > 3 Months & > 6 Months & Delay (mo) \\
\hline Patient delay & 1,433 & $812(56.6)$ & $579(40.4)$ & $401(28.0)$ & $2.00(0.50-10.00)$ \\
\hline Care delay & 1,417 & $222(15.5)$ & $80(5.6)$ & $49(3.5)$ & $0.37(0.20-0.67)$ \\
\hline Consultation-diagnosis & 1,421 & $144(10.0)$ & $66(4.6)$ & $45(3.2)$ & $0.17(0.07-0.37)$ \\
\hline Diagnosis-therapy & 1,427 & $41(2.9)$ & $10(0.7)$ & $3(0.2)$ & $0.13(0.00-0.33)$ \\
\hline Total delay & 1,416 & $1,077(75.1)$ & $732(51.0)$ & $536(37.4)$ & $3.18(1.07-12.17)$ \\
\hline
\end{tabular}

Values are presented as number (\%) or median (25th to 75 th percentile). 


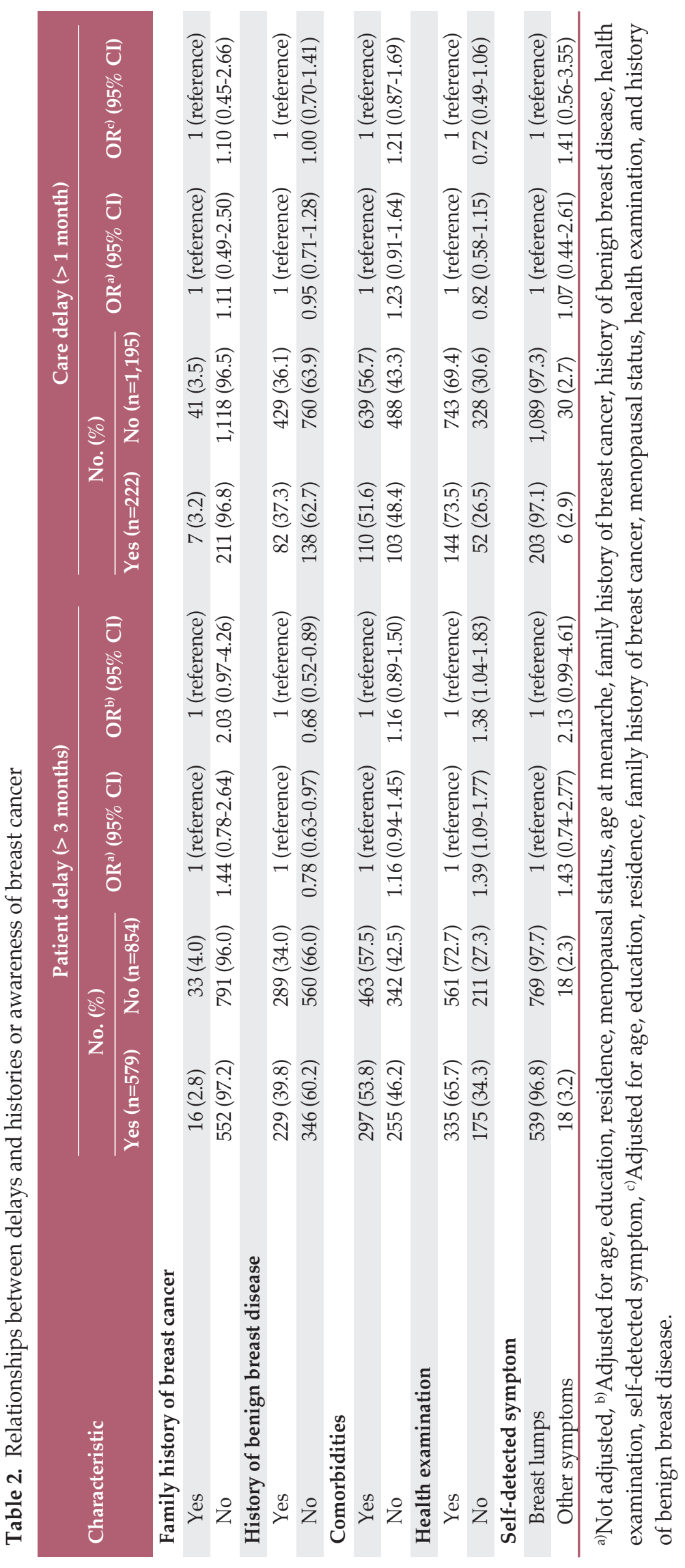

VOLUME51 NUMBER 3 JULY 20191101 


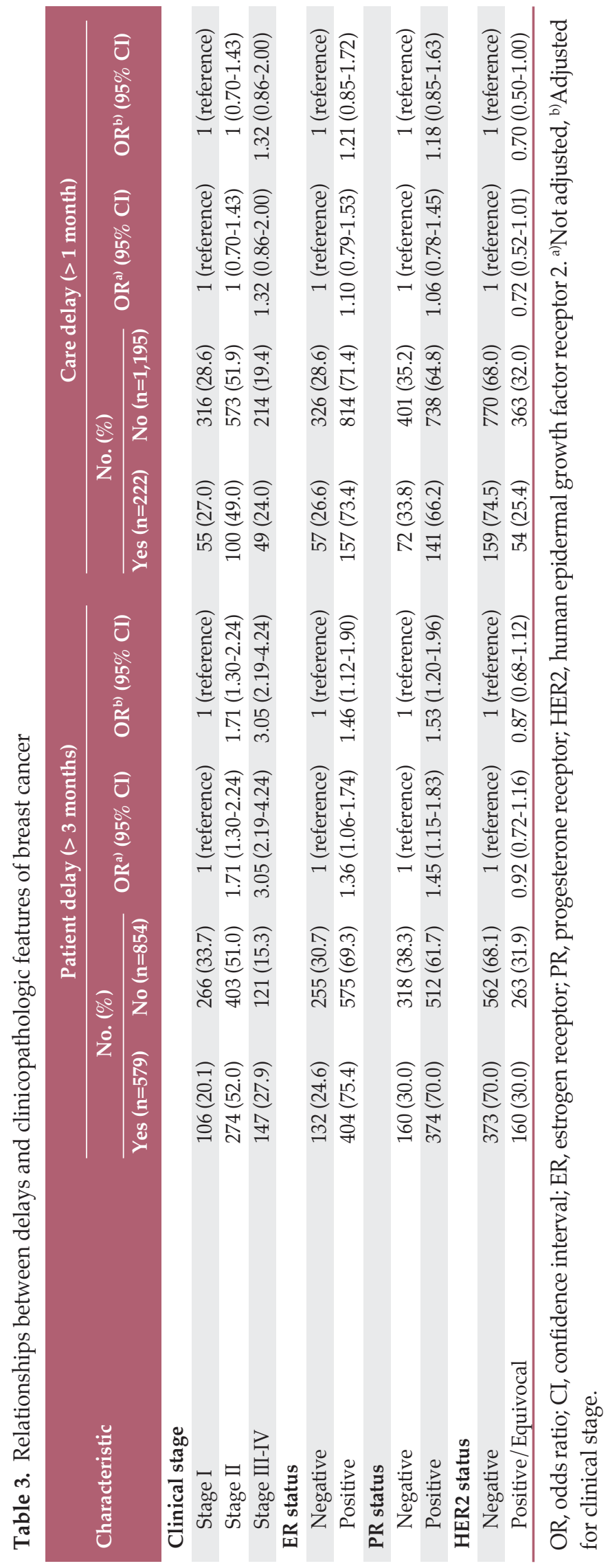


Table 4. Associations of patient and care delays with breast cancer prognosis

\begin{tabular}{|c|c|c|c|c|c|c|}
\hline Prognosis & Delay & No. $(\%)$ & $\mathrm{HR}^{\mathrm{a})}(95 \% \mathrm{CI})$ & $\mathrm{HR}^{\mathrm{b})}(95 \% \mathrm{CI})$ & $\mathrm{HR}^{\mathrm{c}}(\mathbf{9 5} \% \mathrm{CI})$ & $\mathrm{HR}^{\mathrm{d})}(95 \% \mathrm{CI})$ \\
\hline \multirow[t]{6}{*}{ Death } & Patient delay & & & & & \\
\hline & No & $70(9.2)$ & 1 (reference) & 1 (reference) & 1 (reference) & 1 (reference) \\
\hline & Yes & 65 (12.4) & $1.36(0.95-1.95)$ & $1.43(0.98-2.07)$ & $1.17(0.79-1.72)$ & $1.11(0.75-1.66)$ \\
\hline & Care delay & & & & & \\
\hline & No & $114(10.6)$ & 1 (reference) & 1 (reference) & 1 (reference) & 1 (reference) \\
\hline & Yes & $19(9.7)$ & $0.92(0.55-1.54)$ & $0.95(0.56-1.61)$ & $0.85(0.49-1.47)$ & $0.93(0.53-1.61)$ \\
\hline \multirow{6}{*}{$\begin{array}{l}\text { Disease } \\
\text { progression }\end{array}$} & Patient delay & & & & & \\
\hline & No & $118(15.6)$ & 1 (reference) & 1 (reference) & 1 (reference) & 1 (reference) \\
\hline & Yes & 115 (21.9) & $1.43(1.09-1.87)$ & $1.45(1.09-1.91)$ & $1.19(0.89-1.59)$ & $1.17(0.87-1.57)$ \\
\hline & Care delay & & & & & \\
\hline & No & $188(17.5)$ & 1 (reference) & 1 (reference) & 1 (reference) & 1 (reference) \\
\hline & Yes & $42(21.5)$ & $1.33(0.94-1.89)$ & $1.38(0.97-1.97)$ & $1.30(0.91-1.87)$ & $1.37(0.95-1.98)$ \\
\hline
\end{tabular}

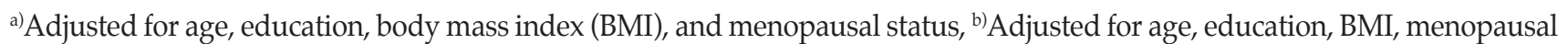
status, and estrogen receptor (ER) status, ${ }^{c}$ Adjusted for age, education, BMI, menopausal status, and clinical stage, ${ }^{\mathrm{d})}$ Adjusted for age, education, BMI, menopausal status, ER status, and clinical stage.

\section{Factors associated with patient and care delays}

The associations of demographic characteristics with patient or care delays were shown in S1 Table. Compared to premenopausal women, postmenopausal women were less likely to experience patient delay (OR, 0.65; 95\% CI, 0.44 to 0.97). Age seemed not impact on patient delay, but it was significantly related to care delay and the women of 41-60 years and $\geq 61$ years had a shorter care delay compared with those aged $\leq 40$ years (OR, $0.60 ; 95 \%$ CI, 0.41 to 0.88 and OR, 0.50 ; $95 \% \mathrm{CI}, 0.25$ to 1.01 , respectively). We did not find any association between other demographic factors and breast cancer delays.

Meanwhile, patient delay but not care delay was associated with history/awareness of diseases as shown in Table 2. Women without a history of benign breast disease had a decreased risk of patient delay compared to those with the history (OR, $0.68 ; 95 \%$ CI, 0.52 to 0.89 ). Women without a history of physical examination were more prone to experience patient delay (OR, 1.38; 95\% CI, 1.04 to 1.83).

We further investigated the relationships between patient/care delays and clinicopathologic features as shown in Table 3. The women with clinical stage I, II, and III-IV reported $28.5 \%, 40.5 \%$, and $54.9 \%$ of patient delay, respectively. Compared to the patients with stage I, the patients with stage II and III-IV had significant increased risks of patient delay (OR, 1.71; 95\% CI, 1.30 to 2.24 and OR, 3.05; 95\% CI, 2.19 to 4.24, respectively). There was also a possible connection between patient delay and positivity of ER (OR, 1.46; 95\% CI, 1.12 to 1.90$)$ or PR (OR, 1.53; 95\% CI, 1.20 to 1.96$)$.

\section{Associations of patient and care delays with prognosis}

We firstly assessed the relationships of demographic and clinicopathologic characteristics with breast cancer outcomes (S1 Table), so as to determine the potential confounders for the analysis of association between patient or care delay and the prognosis of breast cancer. Considering that clinical stage and hormone status may act as intermediate variables between delays and breast cancer prognosis, sensitivity analyses were conducted as shown in Table 4. Before adjustment for clinical stage and ER status, patient delay was significantly associated with a shorter OS and PFS (OS: HR, 1.36; 95\% CI, 0.95 to 1.95 and PFS: HR, 1.43; 95\% CI, 1.09 to 1.87); after adjusted for ER status, the associations stayed almost the same. However, these associations disappeared when adjusted for clinical stage (OS: HR, 1.17; 95\% CI, 0.79 to 1.72 and PFS: HR, 1.19; 95\% CI, 0.89 to 1.59$)$ or both ER status and clinical stage (OS: HR, 1.11; 95\% CI, 0.75 to 1.66 and PFS: HR, $1.17 ; 95 \% \mathrm{CI}, 0.87$ to 1.98$)$. For care delay, there was no significant association with breast cancer prognosis for either OS or PFS.

\section{Discussion}

In the present study, we differentiated the two types of delays for breast cancer in mainland China for the first time and found that patient delay was more often than care delay 
relatively to Western countries. The incidence of patient delay ( $\geq 3$ months) found in the present study $(40.4 \%)$ was much higher than that in America (17\%) [17] and Germany $(18 \%)$ [18], while the incidence of care delay ( $\geq 3$ months) in the present study $(5.6 \%)$ was lower than that in America $(12 \%)[8,17]$ and Germany $(11 \%)$ [4]. This disparity may be able to explain the fact, at least to some extent, that the proportion of clinical stage I among the diagnosed breast cancer patients was around 30\% (year 2005-2007) in China, while this proportion was $45 \%$ in America during the same period [19]. This fact was also supported by another result from the current study that the majority $(93.6 \%)$ of breast cancer subjects detected the initial signs or symptoms by themselves, while only $5.6 \%$ of the patients were exam-detected and $0.8 \%$ of them were imaging-detected, strongly indicating that there was an insufficiency of early screening of breast cancer.

The delay rates seemed vary in developing countries. For example, the median patient and care intervals were 60.5 and 52.5 days (more than $50 \%$ of patient and care delay as defined in the present study), respectively, in Mexico City [20]; almost half the women with breast cancer experienced patient delay ( $>3$ months) in a India rural area [21]; the patient delay (> 3 months) rate was $32.2 \%$ and more than half the patients had care delay (>1 month) in Jordon [22]. Inconsistent specifications of delay types and small samples (around 300 patients, which was apt to unstable results) were probably the main reasons for the differences. The total delay $(>30$ days) rates from the only previous study in China (60.4\%) and the present study $(51.0 \%$ ) (Table 1) were roughly comparable. In addition, interestingly, a previous study showed that patients who had breast cancer had a significantly shorter delay (average, 47.5 days) than those who had a benign breast disease (average, 78.6 days) [23]; unfortunately, there was no similar data in the present study for comparison.

We confirmed that patient delay was associated with advanced clinical stages and poor outcomes of breast cancer, which was intuitive and plausible and consistent with the result in a previous Meta-analysis with more than 100 thousand subjects [2]. As for care delay, we found no significant association with either clinical characteristics or prognosis of breast cancer, which was consistent with the results from the studies by Yoo et al. [5], Brazda et al. [6], and Mujar et al. [7]. This phenomenon may be explained by that the care delay was not long enough to have detrimental effects on the clinical characteristics and survival [24]. It was proposed that very ill patients tended to have the prior medical feedback with a short delay, but their prognosis was always worse [18]; once the patient saw the doctor, further delay might not adversely affect the outcomes, because doctors were able to distinguish between more or less aggressive malignancies $[25,26]$. However, some previous studies did find association between long care delay and worse prognosis of breast cancer $[8,9]$. The reasons of the inconsistency varied, and the inaccuracy of the delay information, such as from cancer registry database, might be the important one [5].

We further found patient delay was associated with premenopausal status, history of benign breast disease, and less physical examination, which was consistent with the results from most of the previous studies [17,27-29]. Women with the history of benign breast disease, who experienced episodes of benign breast alterations, may habitually consider it as the same as before. Similarly, premenopausal women, who had the frequent occurrence of breast pain and hyperplasia, let their vigilance down even if this may be the symptoms of breast cancer and thus caused patient delay [30]. In fact, benign breast diseases mostly occurred among premenopausal (young) women. Therefore, it is plausible that women either with premenopausal status or history of benign breast diseases were more likely to have patients delay. A previous study in Shandong, China, however, indicated that premenopausal status was a risk factor of patient delay, while the history of benign breast disease was a protective factor [13], which was hard to be explained. Nevertheless, elaborating all the suspicious symptoms of breast cancer, particularly to premenopausal women, and emphasizing the probabilities of benign breast disease developing into breast cancer, are of practical significance to help minimize patient delay. The lawsuits and medical dispute profiteers for medical malpractice have been increasing in China [31], though the proportion related to breast cancer is not available. From the data obtained in the United States that breast cancer was the second leading condition involved in malpractice litigation [32], breast cancer is probably one of the main diseases involved in the litigation in China. The results in the present study provided useful information for the practitioners in China to avoid the malpractice.

We would mention a few strength and limitations of the present study. First, the subjects were from 3 large scale teaching hospitals in Guangzhou city and they might be a selected sample of Southern China. However, Guangzhou is the biggest city in Southern China and cancer patients in the adjacent areas, not like other type of diseases, mostly go to the large scale hospitals. Therefore, the present subjects were still quite a representative sample of the breast cancer patients in Southern China. Second, the information about patient and care delays was collected from medical records in a no standard manner. However, it was obtained by the surgeons trusted by the patients who always wished a right decision for their diagnosis and treatment. Moreover, in our pilot investigation, the results from medical records and our interviewers were consistent. Therefore, we believe that our delay data were reliable. Third, the information was collected at the time when patients were newly admitted into hospi- 
tals, and then the recall bias was minimized. Fourth, we had collected and considered as many confounding factors towards the outcomes of breast cancer as possible, and clearly indicated that clinical stage was the mediator in the pathway from patient delay to the prognosis of breast cancer by thorough sensitive analyses. Finally, the delays, particularly patient delay, depend on the patient's knowledge, attitudes, beliefs, and behaviors, for example, complementary and alternative medicine was found to be related to the delays of breast cancer [33], and further studies would be needed to obtain systematic measures for reduction of the delays.

In conclusion, we found that patient delay was more common than care delay in a Southern Chinese breast cancer population, indicating insufficiency of early screening. Patient delay led to a poor prognosis of breast cancer, which was probably mediated by clinical stage. Premenopausal women may be a target population to reduce this delay. Care delay, however, was not associated with any clinical features and outcomes of breast cancer.

\section{Electronic Supplementary Material}

Supplementary materials are available at Cancer Research and Treatment website (https://www.e-crt.org).

\section{Conflicts of Interest}

Conflict of interest relevant to this article was not reported.

\section{Acknowledgments}

This work was supported by the National Natural Science Foundation of China (NO. 81773515).

\section{References}

1. Caplan L. Delay in breast cancer: implications for stage at diagnosis and survival. Front Public Health. 2014;2:87.

2. Richards MA, Westcombe AM, Love SB, Littlejohns P, Ramirez AJ. Influence of delay on survival in patients with breast cancer: a systematic review. Lancet. 1999;353:1119-26.

3. Rabinovich M, Vallejo C, Perez J, Rodriguez R, Cuevas M, Machiavelli $\mathrm{M}$, et al. Impact of delay to treatment upon survival in 1067 patients with breast-cancer. Int J Oncol. 1993;2: 197-201.

4. Arndt V, Sturmer T, Stegmaier C, Ziegler H, Becker A, Brenner $\mathrm{H}$. Provider delay among patients with breast cancer in Germany: a population-based study. J Clin Oncol. 2003;21:1440-6.

5. Yoo TK, Han W, Moon HG, Kim J, Lee JW, Kim MK, et al. Delay of treatment initiation does not adversely affect survival outcome in breast cancer. Cancer Res Treat. 2016;48:962-9.

6. Brazda A, Estroff J, Euhus D, Leitch AM, Huth J, Andrews V, et al. Delays in time to treatment and survival impact in breast cancer. Ann Surg Oncol. 2010;17 Suppl 3:291-6.

7. Mujar M, Dahlui M, Yip CH, Taib NA. Delays in time to primary treatment after a diagnosis of breast cancer: does it impact survival? Prev Med. 2013;56:222-4.

8. Shin DW, Cho J, Kim SY, Guallar E, Hwang SS, Cho B, et al. Delay to curative surgery greater than 12 weeks is associated with increased mortality in patients with colorectal and breast cancer but not lung or thyroid cancer. Ann Surg Oncol. 2013;20:2468-76.

9. Yun YH, Kim YA, Min YH, Park S, Won YJ, Kim DY, et al. The influence of hospital volume and surgical treatment delay on long-term survival after cancer surgery. Ann Oncol. 2012;23: 2731-7.

10. Jones SC, Johnson K. Women's awareness of cancer symptoms: a review of the literature. Womens Health (Lond). 2012;8:57991.

11. Yu W, Yu X, Hu H, Duan G, Liu Z, Wang Y. Use of hospital appointment registration systems in China: a survey study. Glob J Health Sci. 2013;5:193-201.

12. Violence against doctors: why China? Why now? What next? Lancet. 2014;383:1013.

13. Huo Q, Cai C, Zhang Y, Kong X, Jiang L, Ma T, et al. Delay in diagnosis and treatment of symptomatic breast cancer in China. Ann Surg Oncol. 2015;22:883-8.

14. Neal RD, Allgar VL. Sociodemographic factors and delays in the diagnosis of six cancers: analysis of data from the "National Survey of NHS Patients: Cancer". Br J Cancer. 2005;92: 1971-5.

15. Hansen RP, Olesen F, Sorensen HT, Sokolowski I, Sondergaard J. Socioeconomic patient characteristics predict delay in cancer diagnosis: a Danish cohort study. BMC Health Serv Res. 2008;8:49.

16. He JR, Tang LY, Yu DD, Su FX, Song EW, Lin Y, et al. EpsteinBarr virus and breast cancer: serological study in a high-incidence area of nasopharyngeal carcinoma. Cancer Lett. 2011; 309:128-36.

17. Ruddy KJ, Gelber S, Tamimi RM, Schapira L, Come SE, Meyer $\mathrm{ME}$, et al. Breast cancer presentation and diagnostic delays in young women. Cancer. 2014;120:20-5.

18. Arndt V, Sturmer T, Stegmaier C, Ziegler H, Dhom G, Brenner $\mathrm{H}$. Patient delay and stage of diagnosis among breast cancer patients in Germany: a population based study. Br J Cancer. 2002;86:1034-40.

19. Zheng Y, Wu CX, Zhang ML. The epidemic and characteristics of female breast cancer in China. China Oncol. 2013;23:561-9. 
20. Leon-Rodriguez E, Molina-Calzada C, Rivera-Franco MM, Campos-Castro A. Breast self-exam and patient interval associate with advanced breast cancer and treatment delay in Mexican women. Clin Transl Oncol. 2017;19:1276-82.

21. Gangane N, Anshu, Manvatkar S, Ng N, Hurtig AK, San Sebastian M. Prevalence and risk factors for patient delay among women with breast cancer in rural India. Asia Pac J Public Health. 2016;28:72-82.

22. Abu-Helalah AM, Alshraideh HA, Al-Hanaqtah M, Da'na M, Al-Omari A, Mubaidin R. Delay in presentation, diagnosis, and treatment for breast cancer patients in Jordan. Breast J. 2016;22:213-7.

23. Wall P, Moore C, El-Tamer M, Reilly JJ. Diagnostic delay in breast disease: a system analysis of a public urban hospital. Arch Surg. 1998;133:662-6.

24. Hershman D, McBride R, Jacobson JS, Lamerato L, Roberts K, Grann VR, et al. Racial disparities in treatment and survival among women with early-stage breast cancer. J Clin Oncol. 2005;23:6639-46.

25. Gould-Martin K, Paganini-Hill A, Casagrande C, Mack T, Ross RK. Behavioral and biological determinants of surgical stage of breast cancer. Prev Med. 1982;11:429-40.

26. Afzelius P, Zedeler K, Sommer H, Mouridsen HT, BlichertToft M. Patient's and doctor's delay in primary breast cancer: prognostic implications. Acta Oncol. 1994;33:345-51.
27. Ramirez AJ, Westcombe AM, Burgess CC, Sutton S, Littlejohns $\mathrm{P}$, Richards MA. Factors predicting delayed presentation of symptomatic breast cancer: a systematic review. Lancet. 1999;353:1127-31.

28. Nosarti C, Crayford T, Roberts JV, Elias E, McKenzie K, David AS. Delay in presentation of symptomatic referrals to a breast clinic: patient and system factors. Br J Cancer. 2000;82:742-8.

29. Meechan G, Collins J, Petrie KJ. The relationship of symptoms and psychological factors to delay in seeking medical care for breast symptoms. Prev Med. 2003;36:374-8.

30. Schernhammer ES, Laden F, Speizer FE, Willett WC, Hunter DJ, Kawachi I, et al. Rotating night shifts and risk of breast cancer in women participating in the nurses' health study. J Natl Cancer Inst. 2001;93:1563-8.

31. Wang Z, Li N, Jiang M, Dear K, Hsieh CR. Records of medical malpractice litigation: a potential indicator of health-care quality in China. Bull World Health Organ. 2017;95:430-6.

32. Guthrie TH. Breast cancer litigation: an update with practice guidelines. Breast J. 1999;5:335-9.

33. Mohd Mujar NM, Dahlui M, Emran NA, Abdul Hadi I, Wai YY, Arulanantham S, et al. Complementary and alternative medicine (CAM) use and delays in presentation and diagnosis of breast cancer patients in public hospitals in Malaysia. PLoS One. 2017;12:e0176394. 